\title{
High resolution imaging of superficial mosaicity in single crystals using Grazing Incidence Fast Atom Diffraction
}

\section{B. Lalmi, H. Khemliche, A. Momeni, P. Soulisse, and P. Roncin}

Institut des Sciences Moléculaires d'Orsay (ISMO), CNRS - Univ. Paris-Sud 11, F91405 ORSAY Cedex, France

\begin{abstract}
A new table top technique is used to simultaneously analyse the local morphology of crystalline surfaces as well as the misalignment of large scale domains at the topmost surface layer. The approach is based on fast atom diffraction at grazing incidence (GIFAD); the diffraction pattern yields the topology of the surface electronic density, similar to that imaged by Atomic Force Microscopy. If superficial mosaicity is present, diffraction patterns arising from each mosaic domain can be distinguished, providing high sensitivity on the property of each domain. Taking $\mathrm{NaCl}(001)$ as an example, we observe a discrete tilt angle distribution of mosaic domains following an arithmetic progression with a $0.025^{\circ} \pm 0.005$ difference; a twist mosaic angle of $0.09^{\circ} \pm 0.01$ is also observed.
\end{abstract}

Keywords: Diffraction; Fast Atom Diffraction; mosaic structure; atom scattering; particle-surface interactions; ionic insulators 


\section{Introduction}

Actual single crystals always contain defects that induce local distortions to the periodic arrangement. These defects can take several forms: point defects (interstitial atoms, vacancies), linear (dislocations), two dimensional (stacking faults, antiphase boundaries), and three dimensional (amorphous cluster, precipitates) [14]. These defects also induce local modifications in the electronic structure, which can affect considerably the physico-chemical properties [5]. Mosaicity is one of the fundamental defects most often present in single crystals. The concept was first introduced to explain the decrease of intensity in the X-ray reflectivity studies [68]. Mosaic crystals are considered to be formed by a large number of small perfect crystallites slightly disoriented with respect to each other. This is due to an arrangement of dislocations in a pattern separating the crystal into blocks $[9,10]$. The spread of misalignment angles of the crystal blocks gives the degree of mosaicity of the material. Generally, mosaicity can be described in terms of two crystallographic parameters: the out-of plane misorientation called tilt mosaic and the in-plan misorientation called twist mosaic $[11,12]$.

In X-ray diffraction, tilt and twist mosaic are given by the FWHM of symmetric and asymmetric $\omega$-scan rocking curve respectively [13-16]. However, additional structural imperfections and instrumental broadening also affect the linewidth [17-20], imposing measurement for several diffraction orders. Such treatment requires a comparatively high precision and the determined values are often marred by considerable uncertainty. Furthermore, because of the penetration of X-rays in solids, the results are insensitive to the superficial layer, even in grazing geometry which probes a typical depth around $100 \AA$. Local microscopy such as STM or AFM are excellent tools to characterize such crystalline distortion but only in a comparatively reduced scale because atomic resolution cannot be reached on scales larger than few $100 \mathrm{~nm}$ so that superficial mosaicity, is still largely unknown.

In this paper, we present a quantitative characterization of superficial mosaicity, using an exclusively surface sensitive technique, Grazing Incidence Fast Atom Diffraction (GIFAD). This technique is based on the coherent scattering of helium atoms at energies in the $\mathrm{keV}$ range and at grazing incidence angle around $1^{\circ}$. In this geometry, the incident atom energy normal to the surface is below $1 \mathrm{eV}$, thus precluding penetration below the surface.

\section{Experimental details}

All experiments were performed in an ultrahigh vacuum chamber with a base pressure in the $10^{-10}$ Torr range. $\mathrm{He}^{+}$ions are extracted from an ion source at $450 \mathrm{eV}$ energy; they are subsequently neutralized by charge exchange in a He cell. Then two variable apertures are used to reduce the beam divergence and the beam size to less than $1 \mathrm{mrad}$ and $100 \mu \mathrm{m}$ respectively. In the specific experimental conditions used here, the divergence of the direct beam is $0.3 \mathrm{mrad}\left(0.017^{\circ}\right)$. The beam scattered from the sample, and a small fraction of the direct beam, reach a detector located at $\mathrm{L}=865 \mathrm{~mm}$. The latter is of made of microchannel plates and a phosphor screen; images are recorded by a high resolution CCD camera.

In addition to providing the lattice parameter, the intensity distribution within the diffraction pattern reveals the profile of the valence electronic density, thus providing a map of the surface topography comparable to that obtained in AFM but by via reciprocal space technique i.e. with interferometric accuracy, see e.g. [21-23]. The exact beam position, required for an accurate determination of the incident angle, is recorded with the sample surface in a fully retracted position. The commercial $\mathrm{NaCl}(001)$ sample, 10 x $10 \mathrm{~mm}$ in size, was air-cleaved and then rapidly introduced in the vacuum chamber; it was further annealed in UHV at $600^{\circ} \mathrm{C}$. 


\section{Results and discussions}

Figure 1 displays diffraction images obtained under an incidence angle close to 0.9 degree and correspond to a primary beam aligned along [-110] and [100], the two main surface channeling directions. It is worth recalling that GIFAD does not allow for exchange of reciprocal lattice vectors along the beam direction. This is due to the fact that the coupling strength is too weak and the associated energy too high $[22,24]$. As a result, only one Laue circle intercepting the diffraction spots and the direct beam can be observed. Each image contains two types of information, one related to the macroscopic orientation of the surface and the other connected to the microscopic atomic structure.

The peak spacing yields reciprocal vectors of $1.57 \pm 0.02 \AA^{-1}$ and $2.22 \pm 0.02 \AA^{-1}$ which correspond well to the projected lattice parameter along the [-110] and [100] directions of the $\mathrm{NaCl}(001)$ surface respectively. The geometric description of each image can be summarised with three points, the beam position $\mathrm{B}$, the specular spot position $\mathrm{S}$ and the center $\mathrm{C}$ of the Laue circle. The line BS gives the direction of the incidence plane which contains the surface normal while its perpendicular bisector is the projection of the surface plane and contains $\mathrm{C}$. When the beam is perfectly aligned along one of the sample crystallographic axes, C is located on BS.

The intensity of the spots is given by the form factor that, within the Hard-Wall description [25], is simply the modulus of a Fourier-like transform of the corrugation function. Here, the observed intensity is well fitted by a simple cosine corrugation with a half amplitude of $0.29 \AA$, consistent with the value measured with thermal helium scattering [26]. More important, the measured intensity distribution is also extremely sensitive to the angle of incidence (the radius of the Laue circle) and to the misalignment angle between the beam and the crystallographic axis, so that the analysis provides additional evidence of any in plane misalignment.

Despite the small beam size, the grazing geometry results in a beam length on the sample of several millimetres. At $0.9^{\circ}$ incidence, this full length is typically $6 \mathrm{~mm}$, though the actual length is smaller because the sample surface is not fully inserted into the beam, thus a small fraction of the incident beam reaches the detector and provides an angular reference. GIFAD patterns of the same sample but at a different location on the sample surface, recorded under an incidence angle close to $0.5^{\circ}$, are shown in fig. 2 . At this angle, the projected length of the incident He beam onto the surface is nearly twice larger than it is at $0.9^{\circ}$.

The image in fig.2.a was obtained along the [-110] axial surface channelling, it shows an unexpected superposition of diffraction patterns. One can clearly distinguish a series of diffraction spots, forming well defined Laue circles whose centers are aligned in the specular plane (see schematic representation fig.3.a). The different diameters are associated with different effective angles of incidence indicating the presence of different domains within the surface illuminated by the atomic beam. The fact that they appear at different incidence angles indicates that these domains are tilted with a component of the rotation vector located in the surface plane and perpendicular to the beam direction, i.e. parallel to the $\langle 100\rangle$ direction. Similar patterns are observed when the beam is oriented along the [110] axis. The profile through the line in fig.2a is shown in the insert; the multipeak structure is very well described by a regular series of peaks spaced by $0.025^{\circ} \pm 0.005$. We observe similar features on cleaved $\operatorname{KBr}(001)$ samples, with a characteristic tilt angle comparable to the value derived for $\mathrm{NaCl}(001)$.

The image in fig.2.b, recorded with the incident beam along the [100] direction, does not contain any sign of tilted domains with rotation vector parallel to the $\langle 110\rangle$ direction. 
The sensitivity of the diffraction pattern to mosaic structures of the surface is illustrated in fig. 3 . The presence of tilted domains, whether the rotation axis is normal or parallel to the beam, is depicted in fig. $3 \mathrm{a}$ and $3 \mathrm{~b}$ respectively. These schemes describe well the diffraction images of fig. $2 \mathrm{a}$ and $2 \mathrm{~b}$; along the [-110] direction, the fit to the data in fig. 2 a yields $\alpha=0.025^{\circ}$. When the incident beam is parallel to the rotation angle of the tilted domains (see fig. 3b.), Laue circles arising from each domain are simply rotated around the beam position by an amount $\gamma$ equal to the angle between blocks. Then the separation between subsequent spots is given by $\gamma \times D$ where $D$ is the diameter of the Laue circle. Thus rotation angles below one degree would be difficult to resolve. In fact the quasi-gaussian broadening, within the Laue circles, of the diffraction spots observed in fig. 2 could well originate from such a mosaicity. Assuming that images in fig. 1 originate from a single domain and that the extra broadening in fig. 2 is due to rotation of the domains around the incident beam direction, an angular distribution with $1.2^{\circ} \mathrm{FWHM}$ is deduced.

A third situation can be encountered when the atomic beam interacts with twisted mosaic domains; this is shown in fig. 3c. Here the Laue circles arising from the twisted domains are displaced by a distance $d_{\xi}$ proportional to the twist angle. An example of this type of mosaicity is given in fig. 3d, it was recorded on a cleaved $\mathrm{KBr}(001)$ crystal with the beam along the [110] direction.

In the framework of quasi-elastic scattering, which is the case in GIFAD [25], and if we assume that the surface is perfectly flat without defects, diffracted intensity distributions presented in fig. 2 to 4 would not be authorized according to the energy conservation law. Thus such structured intensity distributions could only be related to topographic features of the surface.

Features similar to those shown in fig.2a, with comparable tilt angle values, have been observed by high resolution X-Ray diffraction on cleaved $\mathrm{NaCl}(001)$ [27] and $\mathrm{RbI}$ single crystals [28]. Discrete mosaic distributions have also been reported on thin pseudomorphic $\mathrm{ZnSe}$ films on $\mathrm{GaAs}(001)$, above a critical thickness of $100 \mathrm{~nm}[29,30]$. In the later studies, the tilt angle of the mosaic domains, derived from the positions of satellites spots in SPA-LEED measurements, is found to be $0.2^{\circ}$ with respect to the macroscopic plan, which is only slightly larger than the resolution limit in SPA-LEED. With GIFAD, tilt angles as small as $0.01^{\circ}$ can be measured, limitation comes only from the divergence of the primary beam. The latter can always be improved, but at the expense of a reduced atom flux that would increase the acquisition; the data shown here were typically acquired in 10-100 seconds.

The highly regular distribution of tilted mosaic domains described in fig. 2a is present on a large fraction of the cleaved $\mathrm{NaCl}(001)$ sample surface in the form of patches of mm size, meaning that some macroscopic regions of the surface are nearly free from such defects. In addition to this non-uniform patch distribution, we also observe a strong anisotropy in tilt mosaicity. Along the [-110] and [110] directions, the diffraction images bear the signature of tilted domains that have a finite component of their in-plane rotation vector normal to theses directions. Diffraction images measured along the [100] direction instead show that the domains have no rotation component in this direction. Consequently, we derive that the tilt vector should be parallel to the $\langle 010\rangle$ direction.

On the origin of the observed mosaicity, some information may be gained by comparing results form samples having different shapes. Using the same sample preparation procedure, we clearly observe that round samples yield more mosaicity than square ones. Finally polished $\operatorname{KBr}(001)$ samples, used as a X-ray monochromator window, showed the lowest mosaicity. It is worth mentioning that all these crystals were purchased from the 
same supplier. It is interesting to note that $\mathrm{CaF}_{2}(111)$ samples, cleaved with similar procedures, do not exhibit any measurable mosaicity.

Finally, this uniform distribution of the tilt mosaic domains within patches appears to be a robust characteristic of some ionic crystals and points to some kind of self-organisation of these domains.

An example of a peculiar organization mode is shown in fig. 4. Here, the probe beam is off axis with respect to the main crystallographic directions, exactly $7^{\circ}$ away from the [0-10] direction. The surface corrugation (in terms of valence electron density) as averaged by the beam, is very flat so that only the zeroth-order is observed. As in fig. 2a, a series of equally spaced diffraction spots can be observed, each spot corresponding to specular reflection from a single mosaic domain. A projection along the vertical axis shows two regions, each described by series of peaks equally spaced by $0.027^{\circ}$; however these two regions (marked by I and II in fig. 4) are phase shifted by a half period. Variations induced by moving the beam across the sample surface indicates a main domain size of a few 100 microns, this is consistent with the number of illuminated domains at a given incidence angle.

\section{Conclusions}

In conclusion, we have demonstrated that Grazing Incidence Fast Atom Diffraction (GIFAD) can provide a microscopic as well as long range structural analysis of the top most surface layer. In addition to quantitative information on surface corrugation, it can provide clear signature of surface topographic imperfections. Qualitatively, and quantitatively, we have reported a first experimental characterization of the superficial mosaicity on a $\mathrm{NaCl}(001)$ single crystal. Two types of superficial mosaic structures have been observed; (i) twist mosaic defined by macroscopic blocks lying parallel to the (100) surface but slightly rotated one from another along the [100] direction, and (ii) tilt mosaic domains with a misalignment angle being a multiple of an elementary angle equal to $0.025^{\circ}$.

Because GIFAD is a particularly fast technique than can be operated in situ during thin film growth, it may become a valuable tool to follow in real time the formation dynamics of mosaic domains in constrained multilayered systems.

\section{Acknowledgment}

This work was supported by the ANR under contract number ANR-07-BLAN-0160-01. 


\section{References}

[1] N. D. Mermin, Rev. Mod. Phys. 51, 591, (1979)

[2] G.I. Taylor, J. Inst. Metals. 62, 307, (1938).

[3] J.F.W. Bishop, and R .Hill. Phil Mag, 42, 414, (1951)

[4] A.R. West, Solid State Chemistry and its Applications, John Wiley, New York. (1984)

[5] M. Schulze, R. Reissner, Surf. Sci. 482-485, 285 (2001)

[6] C. G. Darwin.Phil. Mag., 27, 315-657, (1914).

[7] C. G. Darwin. Phil. Mag., 43, 800, (1922).

[8] P. P. Ewald. Ann. Phys. (Leipzig), 54, 519-597, (1917).

[9] M. F. Ashby, Phil. Mag. 21, 399-424, (1970)

[10] A.M. Kosevich, Dislocations in Theory of Elasticity, Naukova dumka, Kiev (1978)

[11] R. Chierchia, T. Böttcher, H. Heinke, S. Einfeldt, S. Figge, and D. Hommel, J. Appl. Phys. 93, 8918, (2003).

[12] R. Chierchia, T. Böttcher, S. Figge, M. Diesselberg, H. Heinke and D.Hommel, Phys. Stat. Sol. (b) 228, No. 2, 403406, (2001)

[13] WL. Chen, YH. Wang, MF. Chen, MF. Huang and JC. Fan, Physica. E. 42 1463-1468, (2010)

[14] H. Heinke, V. Kirchner, S. Einfeldt, and D. Hommel, Appl. Phys. Lett., 77, No. 14, (2000)

[15] V. Srikant, J. S. Speck, and D. R. Clarke, J. Appl. Phys., 82 (9), 4286 (1997)

[16] T. Metzger, R. Höpler, E. Born, O. Ambacher, M. Stutzmann, R. Stömmer,M. Schuster, H. Göbel, S. Christiansen, M. Albrecht, and H. P.Strunk, Phil. Mag. A 77, 1013, (1998)

[17] A. Guinier, Theorie et Technique de la Radiocristallographie (Dunod, Paris, (1964).

[18] M. Fatemi, J. Chaudhuri, J. Mittereder, A.Christou, J. Appl. Phys.,73, 1154, (1993)

[19] P. F. Fewster, X-ray and Neutron Diffraction: Theory and Applications, edited by A. Authier, S. Lagomarsino, and B. K. Tanner, Plenum, New York, (1996)

[20] M. J. Hordon, B.L. Averbach, Acta. Mettall, 9, 237, (1961)

[21] H. Khemliche, P. Rousseau, P. Roncin, V. H. Etgens, and F. Finocchi, Appl. Phys. Lett. 95, 151901, (2009) 
[22] P. Rousseau, H. Khemliche, A. G. Borisov, and P. Roncin, Phys. Rev. Lett. 98, 016104, (2007).

[23] A. Schüller, S. Wethekam, and H. Winter, Phys. Rev. Lett. 98, 016103, (2007).

[24] D. Farias, C. Diaz, P. Nieto, A. Salin, and F. Martin, Chem. Phys. Lett. 390, 250, (2004).

[25] J.R. Manson, H. Khemliche and P.Roncin P Phys. Rev. B. 78, 155408 , (2008).

[26] N. Garcia, G. Armand and J. Lapujoulade, Surface Sci. 68399 (1977)

[27] Yasuharu Kashihara, Shigeru Kimura, Jimpei Harada, Surf. Sci. 214, 477 (1989)

[28] O. Blaschko, G. Ernst and J. R. Schneider, J. Phys. C: Solid State Phys. 10, 23, 1977

[29] W. Weigand, C. Kumpf, M. Sokolowski, A. Bader, C. Schumacher, A. Möginger, W. Faschinger, L. W. Molenkamp, and E. Umbach, phys. stat. sol. (b) 229, 117 (2002).

[30] Christian Kumpf, Wolfgang Weigand, Arthur Müller, Joachim Wagner, Veit Wagner, Peter Bach, Georg Schmidt, Laurens W. Molenkamp, Jean Geurts, Eberhard Umbach, Physica Sstatus Solidi C 4,9 (2007) 


\section{Figure captions}

\section{Figure 1:}

GIFAD patterns recorded with $450 \mathrm{eV}^{4} \mathrm{He}$ atoms on $\mathrm{NaCl}(001)$, under incidence angles of (a) $0.87^{\circ}$ along [-110] and (b) $0.85^{\circ}$ along [100]. The Laue circle passing though the spots is centered on $\mathrm{C}$, intercepts the zeroth order on $\mathrm{S}$ and the direct beam on $\mathrm{B}$ at the lower part of the image. Note that a large fraction of the direct beam is cut to protect the detector.

\section{Figure 2:}

GIFAD patterns recorded with $450 \mathrm{eV}^{4} \mathrm{He}$ atoms on $\mathrm{NaCl}(001)$, under incidence angles of $0.5^{\circ}$ along the [-110] (a) and [100] (b) directions. The insert in (a) shows a line profile and a fit using equally spaced Gaussians.

\section{Figure 3:}

Sensitivity of the diffraction pattern to mosaic defects. Tilt mosaic with a rotation vector in the surface plan, perpendicular (a) and parallel (b) to the beam. Twist mosaic with a rotation vector normal to the surface (c), an experimental example is shown in $(\mathrm{d})$.

\section{Figure 4:}

Diffraction profile measured off axis ( $7^{\circ}$ way from [0-10]); only the zeroth order diffraction spot can be observed (see text). The intensity distribution along the vertical direction indicates two regions of equally spaced peaks, arising from individual tilt mosaic domains separated by $\left.0.055^{\circ} / 2\right)$; these two regions are offset by half spacing. 


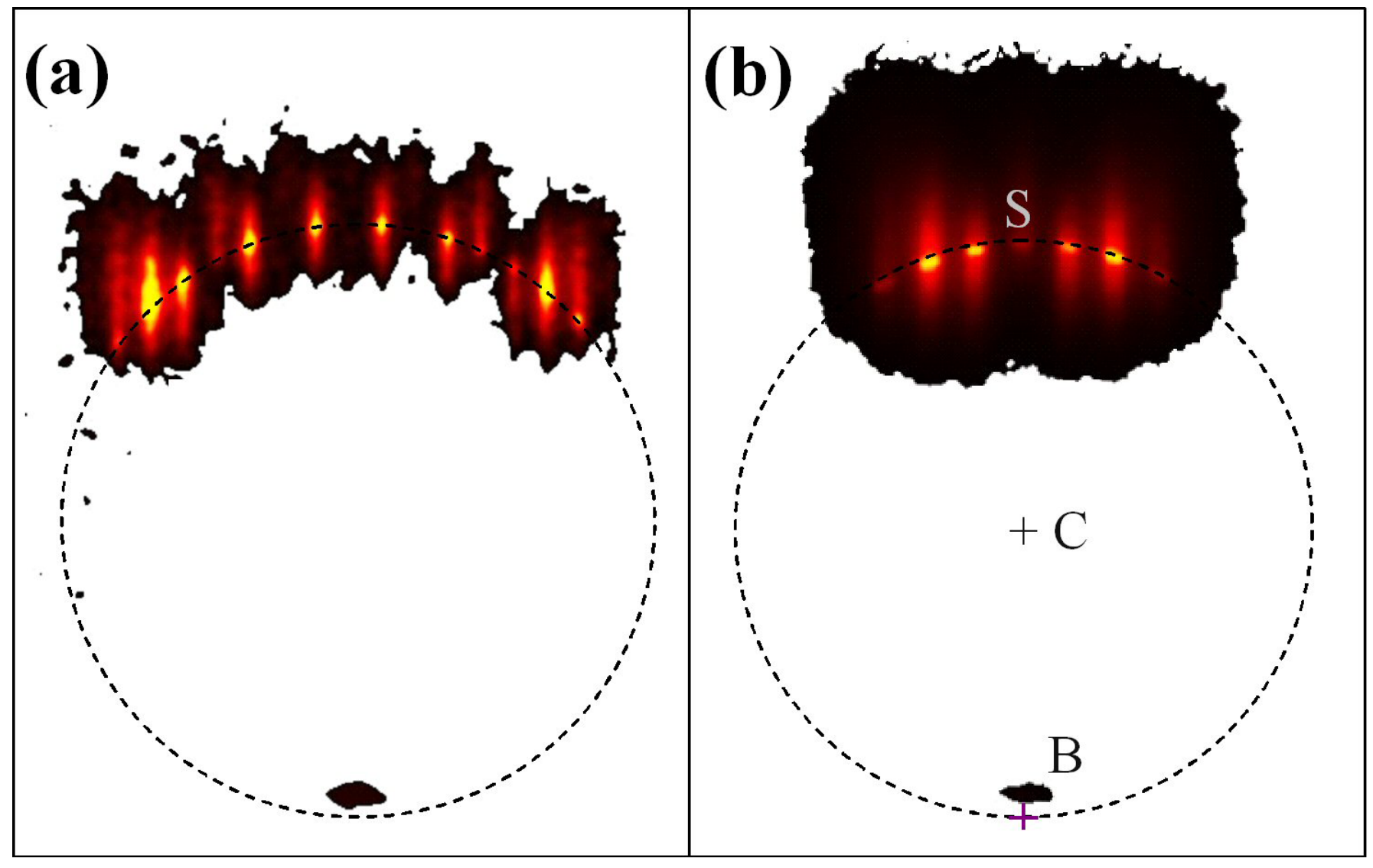

Figure 1 (figure-1.eps) 


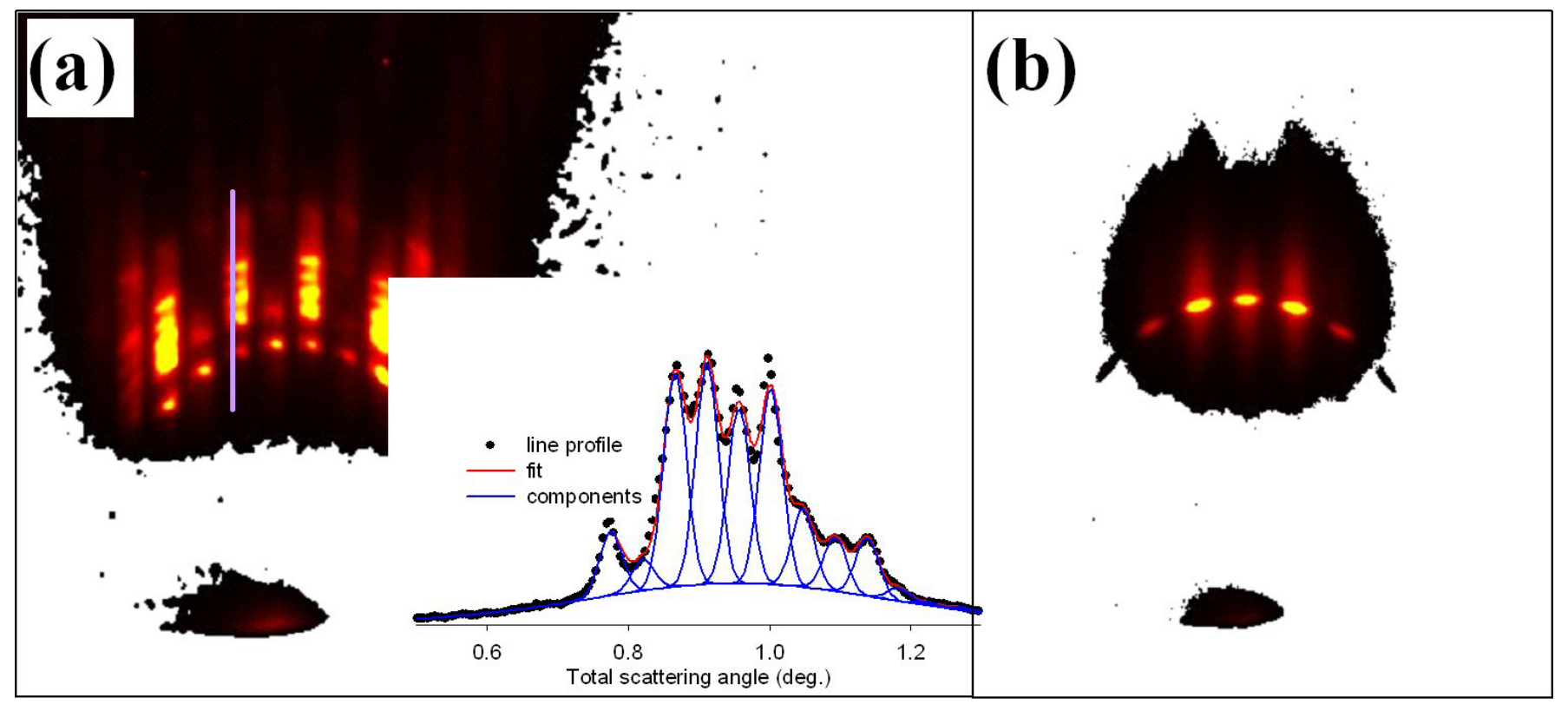

Figure 2 (figure-2.eps) 


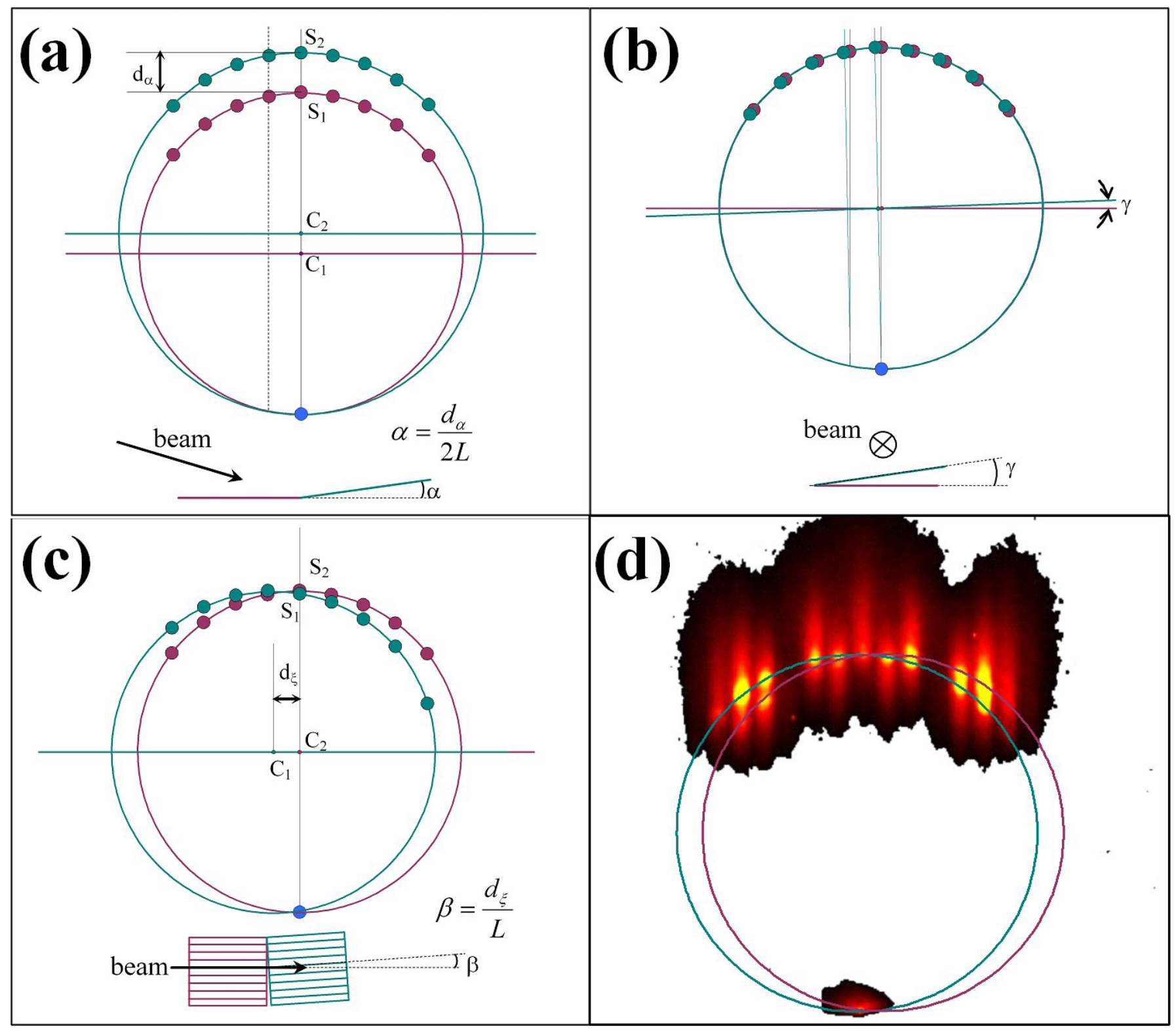

Figure 3 (figure-3.eps) 


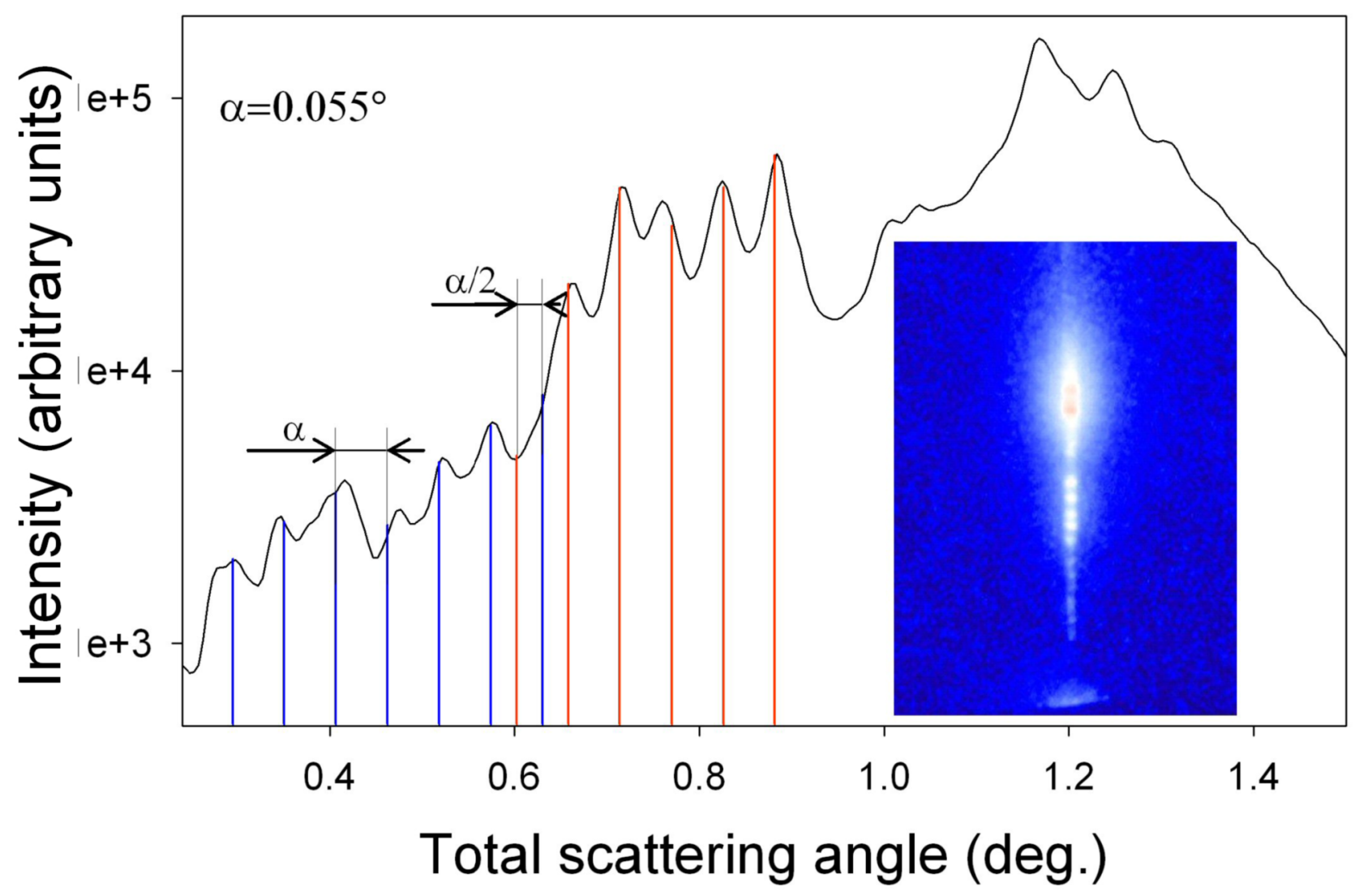

Figure 4 (figure-4.eps) 